\title{
A safety and efficacy trial of artesunate, sulphadoxine-pyrimethamine and primaquine in $P$ falciparum malaria
}

\author{
K L Weerasinghe ${ }^{1}$, G Galappaththy ${ }^{2}$,W P Fernando ${ }^{2}$, D R Wickremasinghe ${ }^{3}$, H M Faizal ${ }^{4}$ and A R Wickremasinghe ${ }^{5}$
}

(Index words: Combination therapy, first line therapy, averting drug resistance)

\begin{abstract}
Objective To determine effectiveness and safety of the combination of artesunate, sulphadoxine+pyrimethamine and primaquine in the treatment of $P$ falciparum malaria.

Design A hospital based prospective study.

Setting Base Hospital, Moneragala.

Methods In 30 P falciparum infected patients admitted to the hospital, blood was taken for estimation of haemoglobin, white cell counts, and serum levels of aspartate aminotransferase, alanine aminotransferase, bilirubin and creatinine. They were administered artesunate, sulphadoxine+ pyrimethamine $(\mathrm{S}+\mathrm{P})$ and primaquine on day 0 (artesunate $4 \mathrm{mg} / \mathrm{kg}$, sulphadoxine $25 \mathrm{mg} / \mathrm{kg}$, pyrimethamine $1.25 \mathrm{mg} /$ $\mathrm{kg}$ and primaquine $0.75 \mathrm{mg} / \mathrm{kg}$ ), and only artesunate on days 1 and 2 (artesunate $4 \mathrm{mg} / \mathrm{kg}$ each day). Blood was examined for malarial parasites, and patients were assessed on days 1, 2, 7, 14, 21 and 28. Patients assessed the severity of selected symptoms. Biochemical analyses were done on day 0 and repeated on days 7 and 28 .

Results Eight patients presented with fever which resolved in 7 patients in 48 hours. Asexual parasites were cleared in $80 \%$ of the 30 patients within 24 hours of treatment and in all 30 by day 7 . Gametocytaemia cleared in all patients by day 14. There were no adverse effects experienced by the patients. The white cell and differential counts, liver enzymes and creatinine levels were within normal limits on all follow up days.
\end{abstract}

Conclusions The combination of artesunate, $\mathrm{S}+\mathrm{P}$ and primaquine was found to be effective and safe in the treatment of uncomplicated $P$ falciparum malaria.

\section{Introduction}

Resistance of malarial parasites to drugs has been defined as "the ability of a parasite strain to multiply or to survive in the presence of concentrations of a drug that normally destroy parasites of the same species or prevent their multiplication" (1). This definition has been modified into relative resistance (yielding to increased doses tolerated by the drug tolerated by the host) or complete resistance (withstanding maximum doses tolerated by the host). Today the term drug resistance usually relates to complete resistance, and is based on parasitological features. In
$P$ falciparum infections failure or success of medication may determine the patient's survival. This is rarely so in malarial infections with the other pathogenic human parasite species. Chloroquine resistance of a high degree with widespread local transmission had been reported in Thailand, Myanmar, Malaysia, Kampuchea, Laos, Vietnam, Philippines and Papua New Guinea (2). Multi-drug resistant strains of malarial parasites have been observed especially in south-east Asian countries such as Myanmar, Thailand China, Laos and Cambodia (3).

Malaria continues to be a major public health problem in Sri Lanka and drug resistance is a major obstacle encountered by the National Malaria Control Programme. $P$ falciparum malaria constitutes a significant number of the malaria cases reported in the country and is responsible for severe disease and mortality.

It is important to discontinue an anti-malarial early when resistance is encountered in a parasite popualtion, deviating from the current practice of continuing to use the first line drug for as long as possible. The high morbidity due to malaria results from the existence of protracted undetected chloroquine resistant $P$ falciparum infections, with few or atypical symptoms, that continue to produce chronic effects such as anaemia (4). A strategy of switching to alternative drugs earlier would reduce the morbidity from protracted infections. This requires active surveillance of chloroquine resistant malaria infections and education of health care providers about the importance of early diagnosis and prompt treatment of chloroquine resistant $P$ falciparum malaria.

Artemisinin, derived from the quinhao (Artemisia anпиа L.) plant and used in China for many centuries is probably the only hope currently available in treatment of drug resistant malaria. Combination of an artemisinin derivative with $\mathrm{S}+\mathrm{P}$ is expected to delay or prevent the emergence of resistance to $\mathrm{S}+\mathrm{P}$. Artemisinin derivatives achieve a substantial and rapid decrease in parasite load but have a short half life; combination with a longer acting drug, such as $\mathrm{S}+\mathrm{P}$, which acts in a different way protects against the emergence of artemisinin resistant parasites (5).

The purpose of this study was to determine if the combination of artesunate, $\mathrm{S}+\mathrm{P}$ and primaquine is safe and effective in the treatment of $P$ falciparum malaria in Sri Lanka. Combination trials done with artesunate and $\mathrm{S}+\mathrm{P}$ in

${ }^{1}$ Base Hospital, Moneragala; ${ }^{2}$ Anti-Malaria Campaign Headquarters, Colombo; ${ }^{3}$ Department of Parasitology, Faculty of Medical Sciences, University of Sri Jayewardenepura, Nugegoda; ${ }^{4}$ Anti-Malaria Campaign, Moneragala; and ${ }^{5}$ Department of Community Medicine and Family Medicine, Faculty of Medical Sciences, University of Sri Jayewardenepura, Nugegoda (Received 10 November 2001, accepted 2 February 2002. Corresponding authors G G and $A R W$ ). 
other countries have shown that it is effective and safe, but primaquine was not used in these studies.

\section{Methodology}

This was a hospital based prospective study done at Base Hospital, Moneragala. Thirty $P$ falciparum infected patients over 15 years of age with a mono-infection and resident within the Moneragala district were included in the study. Patients with one or more general danger signs, any sign of severe malaria, an underlying disease (cardiac, renal, or hepatic disease, malnutrition) or a history of allergy to the drugs used were excluded from the study. On admission a detailed history was taken, a physical examination was done and blood was obtained for estimation of haemoglobin, white cell, count, and serum levels of aspartate aminotransferase, alanine aminotransferase, bilirubin and creatinine. Treatment was administered under direct supervision. Patients were given artesunate (National Medicine Plant Company No. 1 (Mediplants Tex) KM6, Giaiphong Road, Hanoi, Vietnam), $\mathrm{S}+\mathrm{P}$ and primaquine on day 0 (artesunate $4 \mathrm{mg} / \mathrm{kg}$, sulphadoxine $25 \mathrm{mg} / \mathrm{kg}$, pyrimethamine $1.25 \mathrm{mg} / \mathrm{kg}$ and primaquine $0.75 \mathrm{mg} / \mathrm{kg}$ ), and only artesunate on days 1 and 2 (artesunate $4 \mathrm{mg} / \mathrm{kg}$, each day). If the patient vomited within $30 \mathrm{~min}$ of the administration of a drug, it was re-administered. Blood was examined for asexual malarial parasites and gametocytes, and the patients were examined physically and their condition assessed on days 1 and 2. On discharge from the hospital after day 2, patients were requested to visit the hospital on days 7, 14, 21 and 28 of the inital treatment. Medical officers clinically assessed all patients, and blood smear examination for malarial parasites was done on each of the follow up days. Patients assessed the severity of selected symptoms. Biochemical analyses were done on day 0 and repeated on days 7 and 28.

Approval for the study was obtained from the ethical review committee of the Faculty of Medical Sciences, University of Sri Jayewardenepura, and permission to conduct it from the Provincial Health Authority, Uva province.

\section{Results}

Eight patients presented with fever which resolved in $50 \%$ (4 patients) within 24 hours. By 48 hours, fever had resolved in 7 patients. Asexual parasites were cleared in $80 \%$ of all 30 patients within 24 hours of treatment and in $97 \%$ by 48 hours. By the day 7 asexual parsitaemia had cleared in all 30 patients. Gametocytaemia cleared in 3 out of 5 patients by day 7 and in all 30 patients by day 14 . During the 28 day follow up there was no recrudescence of parasitaemia (Table 1)

The white cell and differential counts were within normal limits on all follow up days. There was a small decrease in haemoglobin level on day 7 , but the level returned to the original value by day 28 . The liver enzyme and creatinine levels were within normal limits on all follow up days. Symptomatic treatment was given to patients as appropriate for headache, nausea and vomiting, abdominal pain and diarrhoea.
Table 1. Efficacy of combination therapy

Patients with persisting

\begin{tabular}{llllll} 
Number (\%) & of patients on days \\
\hline 1 & 2 & 7 & 14 & 21 & 28
\end{tabular}

Asexual parasitaemia

$6(20) \quad 1(3) \quad 0 \quad 0000$

Gametocytaemia

$\begin{array}{llllll}5(17) & 4(13) & 2(7) & 0 & 0 & 0\end{array}$

Fever

4 (13) $1(3) \quad 0 \quad 0000$

\section{Discussion}

The current practice of replacing one anti-malarial drug with another once resistance has developed is unsustainable (7). Choosing an alternative drug is often difficult. Few alternatives exist, and those that do are frequently more expensive, or have many more side effects. Sulphadoxine+pyremethamine is the drug most frequently considered in many countries, including Sri Lanka, as the first alternative to chloroquine. $\mathrm{S}+\mathrm{P}$ offers a reasonable combination of easy use, low cost, relatively good tolerance and safety. Nonetheless, it is far from the ideal drug as resistance develops rapidly. There have been a few cases of resistance to $\mathrm{S}+\mathrm{P}$ reported from Sri Lanka (8). These cases may herald the appearance of multi-drug resistant malaria in Sri Lanka as in other south east Asian countries (7).

The results of our study indicate that the combination of artesunate, $\mathrm{S}+\mathrm{P}$ and primaquine is safe and effective in the treatment of $P$ falciparum malaria. The asexual parasitaemia reduced significantly within 24 hours of administration of the drugs on day 0 and cleared by day 7 . The gametocytaemia reduced significantly by 48 hours of treatment. In a study from Gambia (9), in which primaquine was not used, it was found that the mean duration of gametocytaemia was 3 to 4 days in the group which received 3 doses of artesunate as compared to 13 days in the group which received only $\mathrm{S}+\mathrm{P}$, and that gametocytes were found in more than half the children treated with only $\mathrm{S}+\mathrm{P}$. The addition of artesunate either as a 1-day or 3-day course lowered this rate three-fold, as well as gametocyte densities and duration of gametocyte carriage.

In the trials done in Africa using artesunate in combination with $\mathrm{S}+\mathrm{P}$, primaquine was not used as a gametocytocidal drug as the area is holoendemic for malaria and eliminating gametocytes will have little or no impact on transmission. Although artesunate is gametocytocidal, primaquine was included as part of the combination in our trial as malaria in Sri Lanka is of the unstable type, in which transmission can be reduced to a great extent by eliminating the reservoir of infection.

The haemoglobin, alanine aminotransferase, aspartate aminotransferate, creatinine, and bilirubin levels were within normal limites both before and after treatment (Table 2). None of the patients developed clinical signs suggestive of renal or liver disease. Similar results were obtained in the study done in the Gambia (9). None of the patients in our study had to withdraw from it due an adverse reaction or the infection not responding to treatment. These findings indicate that the combination used in this study is safe. 
Table 2. Results of haematological and biochemical investigations

\begin{tabular}{|c|c|c|c|c|c|c|c|}
\hline \multirow[t]{2}{*}{ Investigations } & \multicolumn{2}{|c|}{ Day $0(n=30)$} & \multicolumn{2}{|c|}{ Day $7(n=30)$} & \multicolumn{2}{|c|}{ Day $28(n=30)$} & \multirow[t]{2}{*}{ Normal range } \\
\hline & Mean & $S D$ & Mean & $S D$ & Mean & $S D$ & \\
\hline $\begin{array}{l}\text { White cell } \\
\text { count }(\text { per } \mu / 1)\end{array}$ & 9870 & 1084 & 9454 & 1222 & 9710 & 939 & $4000-11000$ \\
\hline $\mathrm{Hb}(\mathrm{g} / \mathrm{dl})$ & 12.4 & 2.4 & 12.1 & 2.2 & 12.5 & 2.0 & $12-18$ \\
\hline $\operatorname{AST}(\mathrm{u} / \mathrm{l})$ & 28.9 & 8.3 & 36.2 & 15.5 & 30.8 & 9.3 & $10-35$ \\
\hline $\operatorname{ALT}(\mathrm{u} / \mathrm{l})$ & 27.0 & 8.8 & 42.1 & 32.7 & 30.1 & 14.4 & $10-35$ \\
\hline S. bilirubin (mg/dl) & 0.8 & 0.6 & 0.8 & 0.8 & 0.8 & 0.3 & $0.2-1.0$ \\
\hline S. creatinine $(\mathrm{mg} / \mathrm{dl})$ & 1.4 & 1.5 & 1.1 & 0.3 & 1.1 & 0.2 & $0.7-1.7$ \\
\hline
\end{tabular}

The symptoms experienced by patients (Table 1) following combination therapy with artesunste, $\mathrm{S}+\mathrm{P}$ and primaquine appear to be due to the malaria infection per se rather than an adverse reaction to a drug or to the combination, as they were present at the time of diagnosis and subsided with treatment. The absence of other serious drug related adverse effects in several populations that have been subjected to combination therapy indicate that it is safe and well tolerated in the usual doses $(6,7,9)$.

Malarial parasites acquire resistance to antimalarial drugs through mutation. Rapid parasite clearance is expected to reduce the chances of the emergence of drug resistant strains (10). Artemisinin derivatives are the most potent and rapidly acting antimalarial drugs available (5). A combination of a short acting artemisinin derivative with a longer acting drug, such as $\mathrm{S}+\mathrm{P}$, has the advantage that parasites remaining after the action of the artemisinin derivative has ceased are eliminated by a drug with a different mode of action, and are no longer exposed to the atremisinin. Theoretically, emergence of resistance to each drug is less likely when the drugs are combined.

It is estimated that over $50 \%$ of $P$ falciparum infections in Sri Lanka are resistant to chloroquine. WHO recommends that drug policy should be changed when over $25 \%$ of $P$ falciparum infections are resistant to first line therapy. Hence it is imperative that a review of the present drug policy is made, and the Anti-Malaria Campaign is now looking at the different options that are available in Sri Lanka.

$\mathrm{S}+\mathrm{P}$ is the recommended second line of treatment for chloroquine resistant falciparum malaria. As resistance to $\mathrm{S}+\mathrm{P}$ has not been reported widely as yet, the addition of an anti-malarial such as artesunate that is quickly eliminated from the body will help in averting the predicted disaster of $\mathrm{S}+\mathrm{P}$ resistance developing rapidly, if it is used alone as the first line of treatment, as has already been reported from other countries. The combination of artesunate, $\mathrm{S}+\mathrm{P}$ and primaquine should be considered as first line therapy for $P$ falciparum malaria in Sri Lanka.

\section{Acknowledgements}

We acknowledge with thanks the support given by the Deputy Provincial Director of Health Services, Moneragala, the Director, Base Hospital, Moneragala, and staff of the medical ward, the hospital laboratory and the Anti-Malaria Campaign, Moneragala.

\section{References}

1. Wernsdorfer WH. The development and spread of drug resistant malaria. Parasitology Today 1991; 7: 297-302.

2. Black RH, Canfield CJ, Clyde DF, Peters W, Wernsdorfer WH. Drug resistance in malaria. In Bruce-Chwatt LJ ed. Chemotherapy of malaria. 2nd ed. Geneva, WHO, 1981; 105-9.

3. World Health Organisation. Assessment of therapeutic efficacy of antimalarial drugs for uncomplicated falciparum malaria. 1997; 16.

4. Alles HA, de Silva D, Mendis KN. Atypical presentation of malaria associated with chloroquine resistance. Ceylon Medical Journal 1995; 40: 45.

5. White NJ, Nosten F, Looaresuwan S, et al. Averting a malaria disaster. Lancet 1999; 353: 1965-7.

6. Gordi T. Clinical pharmacokinetics of the antimalarial artimisinin based on saliva sampling. Scts Universitatis Upsaliensis. Comprehensive summaries of Uppsala dissertations from the Faculty of Pharmacy, 2001; 11-3.

7. White NJ, Oliaro PL. Rationale for combination therapy for malaria. Parasitology Today 1996; 12: 399-401.

8. Handunetti SM, Jayasinghe S, Pathirana PPSL, Fernando R, Sheriff MHR, Mendis KN. Sulphadoxine-pyrimethamine and chloroquine-resistant $P$ falciparum in Sri Lanka. Ceylon Medical Journal 1994; 39: 45-6.

9. Seidlein LV, Milligan P, Pinder M, Bojang K, Anyalebechi C, et al. Efficacy of artesunate plus pyrimethamine+sulphadoxine for uncomplicated malaria in Gambian children: a double-bind, randomised, controlled trial. Lancet 2000; 355: 352-7.

10. White NJ. Drug resistance in malaria. British Medical Bulletin 1998; 54: 703-15.

11. Galappaththy G. A study of chloroquine resistant $P$ falciparum malaria in Sri Lanka. MD Thesis, University of Colombo, 2001. 